\title{
Identification of potential diagnostic and prognostic biomarkers for prostate cancer
}

\author{
QIANG ZHANG ${ }^{1 *}$, XIUJUAN YIN $^{1 *}$, ZHIWEI PAN $^{2}$, YINGYING CAO $^{3}$, SHAOJIE HAN $^{4}$, \\ GUOJUN GAO ${ }^{5}$, ZHIQIN GAO ${ }^{1}$, ZHIFANG PAN ${ }^{1}$ and WEIGUO FENG ${ }^{1}$ \\ ${ }^{1}$ College of Bioscience and Technology, Weifang Medical University, Weifang, Shandong 261053; \\ ${ }^{2}$ Department of Medicine,Laizhou Development Zone Hospital, Yantai, Shandong 261400; \\ ${ }^{3}$ College of Clinical Medicine, Weifang Medical University; ${ }^{4}$ Changle County Bureau of Animal Health and Production; \\ ${ }^{5}$ Urology Department, Affiliated Hospital of Weifang Medical University, Weifang, Shandong 261053, P.R. China
}

Received April 4, 2019; Accepted July 25, 2019

DOI: 10.3892/ol.2019.10765

\begin{abstract}
Prostate cancer (PCa) is one of the most common malignant tumors worldwide. The aim of the present study was to determine potential diagnostic and prognostic biomarkers for PCa. The GSE103512 dataset was downloaded, and the differentially expressed genes (DEGs) were screened. Gene Ontology (GO), Kyoto Encyclopedia of Genes and Genomes (KEGG) and protein-protein interaction (PPI) analyses of DEGs were performed. The result of GO analysis suggested that the DEGs were mostly enriched in 'carboxylic acid catabolic process', 'cell apoptosis', 'cell proliferation' and 'cell migration'. KEGG analysis results indicated that the DEGs were mostly concentrated in 'metabolic pathways', 'ECM-receptor interaction', the 'PI3K-Akt pathway' and 'focal adhesion'. The PPI analysis results showed that Golgi membrane protein 1 (GOLM1), melanoma inhibitory activity member 3 (MIA3), ATP citrate lyase (ACLY) and G protein subunit $\beta 2$ (GNB2) were the key genes in PCa, and the Module analysis revealed that they were associated with 'ECM-receptor interaction', 'focal adhesion', the 'PI3K-Akt pathway' and the 'metabolic pathway'. Subsequently, the gene expression was confirmed using Gene Expression Profiling Interactive Analysis and the Human Protein Atlas. The results demonstrated that GOLM1 and ACLY expression was significantly upregulated $(\mathrm{P}<0.05)$ in PCa compared with that in normal tissues. Receiver operating characteristic and survival analyses were performed. The results showed that area under the curve values of these genes all exceeded 0.85 , and high expression of these genes
\end{abstract}

Correspondence to: Dr Weiguo Feng or Professor Zhifang Pan, College of Bioscience and Technology, Weifang Medical University, 7166 Baotong Street, Weifang, Shandong 261053, P.R. China

E-mail: fengwg@wfmc.edu.cn

E-mail: sdwfpzf@126.com

${ }^{*}$ Contributed equally

Key words: biomarker, prostate cancer, bioinformatics analysis, differentially expressed gene, identification was associated with poor survival in patients with $\mathrm{PCa}$. In conclusion, this study identified GOLM1 and ACLY in PCa, which may be potential diagnostic and prognostic biomarker of PCa.

\section{Introduction}

Prostate cancer ( $\mathrm{PCa}$ ) is the most common cancer worldwide, and its incidence rate has increased continuously in recent years; the number of new cases of $\mathrm{PCa}$ worldwide increased from 1.11 million in 2015 to 1.28 million in 2018 (1-3). It has been estimated that $>1$ million men are diagnosed and $>300,000$ succumb to the disease annually (4). Although radical prostatectomy is an effective treatment, early detection of PCa is difficult (2). Therefore, early diagnosis is extremely important for PCa treatment. At present, prostate-specific antigen (PSA) is used as a biomarker for PCa diagnosis (5). however, this method has numerous defects. For example, the specificity is low when PSA is moderately elevated $(6,7)$. As a result, identification of more specific biomarkers is essential in order to detect patients at an early stage of $\mathrm{PCa}$ and to provide patients with an optimal treatment.

Microarrays are an efficient tool for analysis of differentially expressed genes (DEGs) and could be applied to identify potential biomarkers for the diagnosis and prognosis of cancer $(8,9)$. During the past decade, various DEGs in colorectal cancer and pancreatic carcinoma have been identified using microarrays $(10,11)$. However, the results indicated that these biomarkers are not enough for the diagnosis and prognosis of $\mathrm{PCa}$, and no reliable biomarker was validated for clinical use (12). Therefore, potential diagnostic and prognostic biomarkers need to be further identified using microarrays and bioinformatics.

The aim of the present study was to determine potential diagnostic and prognostic biomarkers of PCa. Firstly, the GSE103512 dataset was analyzed and the DEGs were screened. Secondly, Gene Ontology (GO), Kyoto Encyclopedia of Genes and Genomes (KEGG) and protein-protein interaction (PPI) analyses of DEGs were performed. The expression of key genes was verified using Gene Expression Profiling 
Interactive Analysis (GEPIA) and Human Protein Atlas (HPA) analysis. Finally, receiver operating characteristic (ROC) and survival analyses were performed to evaluate the diagnostic and prognostic value of these genes.

\section{Materials and methods}

Differential expression analysis. The mRNA expression profile of the GSE103512 dataset was downloaded from Gene Expression Omnibus (GEO) (http://www.ncbi.nlm.nih. gov/geo/). The dataset included 60 PCa samples and 7 normal prostate samples (13). The expression profile of GSE103512 was subsequently analyzed using Morpheus online tool (https://software.broadinstitute.org/morpheus/) (14). A classical t-test was used to identify the DEGs between PCa and normal prostate tissue. A fold-change $>2$ and $\mathrm{P}<0.05$ were considered to indicate a statistically significant difference.

GO and KEGG pathway analysis. To characterize the function and pathway of DEGs, GO and KEGG pathway analyses were performed using the Database for Annotation, Visualization and Integrated Discovery (https://david.ncifcrf.gov/) (9). $\mathrm{P}<0.05$ was considered as statistically significant.

PPI network and module analysis. To assess the interactive associations among DEGs, the DEGs were analyzed using the Search Tool for the Retrieval of Interacting Genes/Proteins database, and a score $>0.4$ was considered significant (15). Subsequently, PPI network was built by the Cytoscape software (version 3.3.0) (16). Finally, the modules were selected using the plug-in Molecular Complex Detection (MCODE), and the pathway analysis was conducted in the modules. $\mathrm{P}<0.05$ was considered as significant (16).

Validation of gene expression. In this study, GEPIA (http://gepia.cancer-pku.cn/) was used to verify the reliability of the mRNA expression of genes (17). HPA database (https://www.proteinatlas.org/) was applied to confirm the protein expression of genes between PCa and normal prostate tissues based on immunohistochemistry (IHC) (17).

ROC analysis and survival analysis. To assess the sensitivity and specificity of the key genes for PCa diagnosis, ROC curves were created by GraphPad Prism software (version 7.0, GraphPad Software, Inc.), based on the data of GSE103512, and the area under the curve (AUC) was used to evaluate the ROC effect (11). In addition, GEPIA was used to further verify the prognostic value of the genes (18).

\section{Results}

Identification of DEGs. A total of 2,000 DEGs were screened from GSE103512, including 1,000 upregulated and 1,000 downregulated genes, comparing between PCa and normal prostate tissues. The heat map, including the top 40 downregulated and 40 upregulated genes, is presented in Fig. 1.

$G O$ and KEGG pathway analysis. To delineate the function of the DEGs, GO and KEGG pathway analyses were conducted. The results of GO analysis indicated that the DEGs were mostly enriched in biological processes, including 'carboxylic acid catabolic process', 'negative regulation of cell death', 'apoptotic process', 'cell proliferation' and 'cell migration' (Table I). For cell component, the DEGs were mostly concentrated in 'Golgi apparatus part', 'endoplasmic reticulum', 'extracellular matrix', 'receptor complex' and 'anchoring junction'. For molecular function, the DEGs were mainly concentrated in 'coenzyme binding', 'transferase activity', 'transferring acyl groups', 'calcium ion binding' and 'phosphatidylserine binding'. In addition, the KEGG analysis results suggested that the DEGs were mostly concentrated in 'metabolic pathways', 'ECM-receptor interaction', the 'PI3K-Akt pathway', 'pathways in cancer' and 'focal adhesion' (Table II).

PPI analysis of DEGs. A total of four key genes with high degrees of interaction were selected using the Cytoscape software. The key genes included Golgi membrane protein 1 (GOLM1), melanoma inhibitory activity member 3 (MIA3), ATP citrate lyase (ACLY) and G protein subunit $\beta 2$ (GNB2). Furthermore, the top three modules were obtained using plug-ins MCODE analysis (Fig. 2). The results of enrichment analysis revealed that the genes in the modules were associated with 'ECM-receptor interaction', 'focal adhesion', 'PI3K-Akt pathway', 'PPAR pathway', 'AMPK pathway', 'metabolic pathways', 'pathways in cancer', 'gap junction' and 'cell cycle' (Table III).

Validation of key genes expression. The expression of key genes was identified by GEPIA and HPA analysis. The results of GEPIA analysis suggested that the mRNA expression of MIA3 and GNB2 was slightly upregulated in $\mathrm{PCa}(\mathrm{P}>0.05)$, and the expression of GOLM1 and ACLY was upregulated significantly $(\mathrm{P}<0.05)$. In addition, IHC results from HPA showed that GOLM1 and ACLY expression was upregulated significantly in PCa compared with that in normal prostate tissues (Fig. 3).

Identification of key genes for diagnosis and prognosis of $P C a$. To evaluate the potential efficiency of these four genes as diagnostic biomarkers, ROC curves were prepared. AUC values of GOLM1, MIA3, ACLY and GNB2 were $>0.85$, suggesting that these four genes had high sensitivity and specificity for $\mathrm{PCa}$ diagnosis (Fig. 4A-D). These results indicated that GOLM1, MIA3, ACLY and GNB2 may be used as biomarkers for the diagnosis of $\mathrm{PCa}$.

Survival analysis of the key genes conducted using GEPIA data. The results demonstrated that GOLM1 and ACLY were significantly associated with the prognosis of patients $(\mathrm{P}<0.05)$, and high expression of these genes was associated with a poor prognosis (Fig. 4E-H). The data suggested that GOLM1 and ACLY may serve as candidate biomarkers for the prognosis of $\mathrm{PCa}$.

\section{Discussion}

$\mathrm{PCa}$ is the most prevalent cancer in males, and an early diagnosis is difficult $(1,2)$. Therefore, novel diagnostic and 

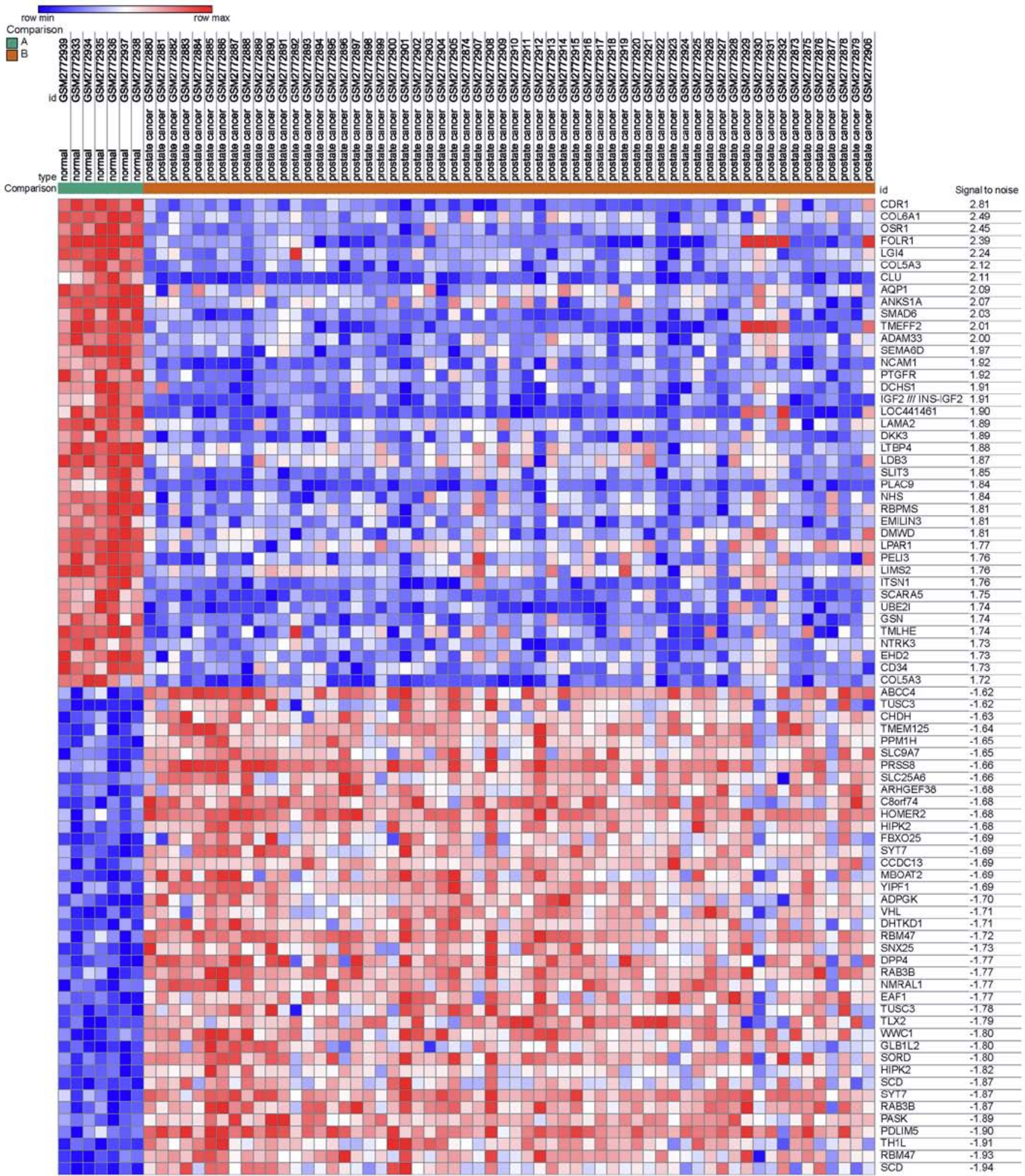

Figure 1. Heat map of the top 80 differentially expressed genes, including 40 upregulated genes and 40 downregulated genes. Red represents upregulated genes and blue represents downregulated genes.

prognostic biomarkers need to be further examined. In the present study, GSE103512 was downloaded and 2,000 DEGs were screened using bioinformatics analysis. The results of GO and KEGG analyses revealed that the DEGs were mostly enriched in 'carboxylic acid catabolic process', 'cell apoptosis', 'cell proliferation' and 'cell migration'. PPI analysis indicated that GOLM1, MIA3, ACLY and GNB2 were the key genes with high degrees. The results of GEPIA and HPA analysis showed the expression of GOLM1 and ACLY was upregulated in PCa tissues. ROC analysis and survival analysis suggested that GOLM1 and ACLY may be used as biomarkers for the diagnosis and prognosis of PCa. 
Table I. GO annotation of differentially expressed genes in prostate cancer.

A, Upregulated genes

\begin{tabular}{|c|c|c|c|}
\hline Category & Term/gene function & Count & P-value \\
\hline GOTERM_BP_FAT & Carboxylic acid catabolic process & 14 & $2.3 \times 10^{-6}$ \\
\hline GOTERM_BP_FAT & Organic acid catabolic process & 14 & $1.0 \times 10^{-5}$ \\
\hline GOTERM_BP_FAT & Oxoacid metabolic process & 28 & $2.0 \times 10^{-5}$ \\
\hline GOTERM_BP_FAT & Cellular amino acid metabolic process & 14 & $3.5 \times 10^{-5}$ \\
\hline GOTERM_BP_FAT & Small molecule catabolic process & 15 & $2.2 \times 10^{-4}$ \\
\hline GOTERM_CC_FAT & Golgi apparatus part & 34 & $4.5 \times 10^{-7}$ \\
\hline GOTERM_CC_FAT & Endoplasmic reticulum & 42 & $2.1 \times 10^{-4}$ \\
\hline GOTERM_CC_FAT & Membrane-bounded vesicle & 74 & $3.0 \times 10^{-4}$ \\
\hline GOTERM_CC_FAT & Extracellular exosome & 60 & $5.9 \times 10^{-4}$ \\
\hline GOTERM_CC_FAT & Cell junction & 29 & $3.0 \times 10^{-2}$ \\
\hline GOTERM_MF_FAT & Coenzyme binding & 11 & $2.0 \times 10^{-4}$ \\
\hline GOTERM_MF_FAT & Transferase activity, transferring acyl groups & 10 & $1.0 \times 10^{-2}$ \\
\hline GOTERM_MF_FAT & Secondary active transmembrane transporter activity & 9 & $1.4 \times 10^{-2}$ \\
\hline GOTERM_MF_FAT & Ligase activity & 12 & $2.3 \times 10^{-2}$ \\
\hline GOTERM_MF_FAT & Cadherin binding & 9 & $5.6 \times 10^{-2}$ \\
\hline
\end{tabular}

B, Downregulated genes

\begin{tabular}{|c|c|c|c|}
\hline Category & Term/gene function & Count & P-value \\
\hline GOTERM_BP_FAT & Negative regulation of cell death & 21 & $1.5 \times 10^{-6}$ \\
\hline GOTERM_BP_FAT & Apoptotic process & 28 & $1.9 \times 10^{-6}$ \\
\hline GOTERM_BP_FAT & Cell proliferation & 30 & $2.0 \times 10^{-6}$ \\
\hline GOTERM_BP_FAT & Regulation of signal transduction & 39 & $5.3 \times 10^{-6}$ \\
\hline GOTERM_BP_FAT & Cell migration & 20 & $8.7 \times 10^{-4}$ \\
\hline GOTERM_CC_FAT & Extracellular matrix & 14 & $3.9 \times 10^{-5}$ \\
\hline GOTERM_CC_FAT & Receptor complex & 8 & $2.2 \times 10^{-2}$ \\
\hline GOTERM_CC_FAT & Anchoring junction & 9 & $4.5 \times 10^{-2}$ \\
\hline GOTERM_CC_FAT & Cell surface & 11 & $5.8 \times 10^{-2}$ \\
\hline GOTERM_CC_FAT & Adherens junction & 8 & $8.4 \times 10^{-2}$ \\
\hline GOTERM_MF_FAT & Calcium ion binding & 14 & $9.3 \times 10^{-4}$ \\
\hline GOTERM_MF_FAT & Phosphatidylserine binding & 4 & $1.4 \times 10^{-3}$ \\
\hline GOTERM_MF_FAT & Ion binding & 33 & $5.5 \times 10^{-2}$ \\
\hline GOTERM_MF_FAT & Receptor activity & 19 & $5.7 \times 10^{-2}$ \\
\hline GOTERM_MF_FAT & Molecular transducer activity & 19 & $5.7 \times 10^{-2}$ \\
\hline
\end{tabular}

GO, Gene Ontology; BP, biological process; CC, cell component; MF, molecular function.

A

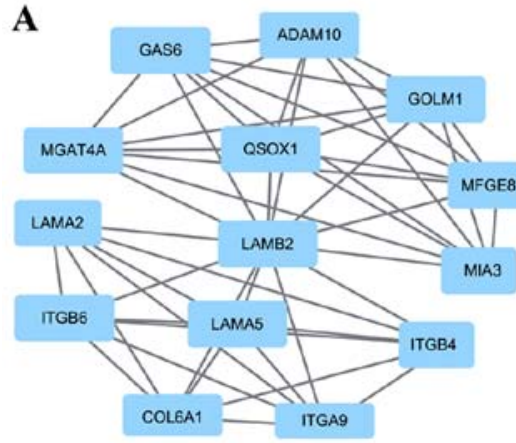

B

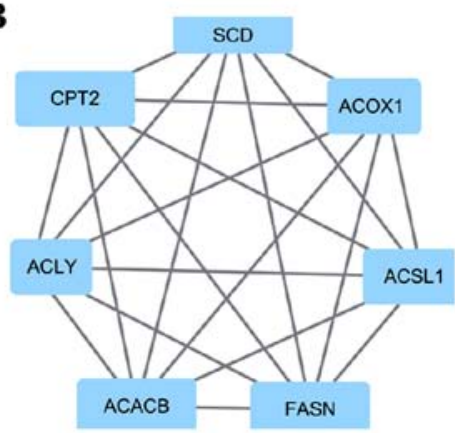

C

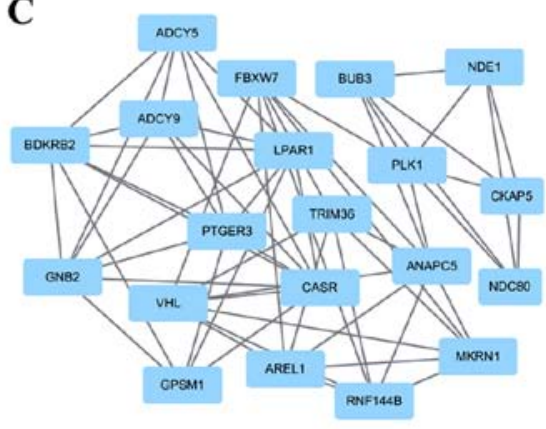

Figure 2. Top three modules from the protein-protein interaction network. (A) Module 1; (B) Module 2; (C) Module 3. 
Table II. KEGG pathway analysis of differentially expressed genes in prostate cancer.

A, Upregulated genes

\begin{tabular}{|c|c|c|c|}
\hline KEGG term & Count & P-value & Genes \\
\hline Metabolic pathways & 39 & $2.5 \times 10^{-6}$ & $\begin{array}{l}\text { AGPAT3, DHCR24, ABAT, DHCR7, ADPGK, ACLY, } \\
\text { CDS1, GMDS, NANS, UAP1, ACACB, ADI1, ACAD8, } \\
\text { ACSL1, ALDH6A1, AKR1A1, CRLS1, CHDH, DCXR, } \\
\text { DDOST, FASN, GCNT2, GLUD2, HGD, MGAT4A, } \\
\text { MOGS, MBOAT2, MCCC2, MTMR3, PLA2G12A, } \\
\text { PAFAH1B3, GALNT7, RDH11, SORD, SAT1, SMS, } \\
\text { SYNJ2, TALDO1, TUSC3 }\end{array}$ \\
\hline Amino sugar and nucleotide sugar metabolism & 4 & $3.5 \times 10^{-2}$ & GMDS, NANS, UAP1, PGM3 \\
\hline Protein processing in endoplasmic reticulum & 7 & $4.1 \times 10^{-2}$ & $\begin{array}{l}\text { SEC13, SEC24A, XBP1, DDOST, MOGS, MAPK9, } \\
\text { TUSC3 }\end{array}$ \\
\hline Glycerophospholipid metabolism & 5 & $5.4 \times 10^{-2}$ & AGPAT3, CDS1, CRLS1, MBOAT2, PLA2G12A \\
\hline Adipocytokine signaling pathway & 4 & $8.7 \times 10^{-2}$ & ACACB, ACSL1, CAMKK2, MAPK9 \\
\hline
\end{tabular}

B, Downregulated genes

\begin{tabular}{|c|c|c|c|}
\hline KEGG term & Count & P-value & Genes \\
\hline ECM-receptor interaction & 8 & $9.2 \times 10^{-6}$ & $\begin{array}{l}\text { COL4A5, COL5A3, COL6A1, HSPG2, ITGB4, LAMA2, } \\
\text { LAMA5, TNXB }\end{array}$ \\
\hline PI3K-Akt signaling pathway & 12 & $1.3 \times 10^{-4}$ & $\begin{array}{l}\text { ANGPT1, COL4A5, COL5A3, COL6A1, FGF10, FGFR1, } \\
\text { ITGB4, LAMA2, LAMA5, LPAR1, PTEN, TNXB }\end{array}$ \\
\hline Pathways in cancer & 11 & $1.8 \times 10^{-3}$ & $\begin{array}{l}\text { ADCY5, COL4A5, FGF10, FGFR1, LAMA2, LAMA5, } \\
\text { LPAR1, PTEN, PML, PTGER3, TCF7L1 }\end{array}$ \\
\hline Focal adhesion & 8 & $2.0 \times 10^{-3}$ & $\begin{array}{l}\text { COL4A5, COL5A3, COL6A1, ITGB4, LAMA2, LAMA5, } \\
\text { PTEN, TNXB }\end{array}$ \\
\hline Regulation of lipolysis in adipocytes & 4 & $1.2 \times 10^{-2}$ & ADCY5, NPR1, PTGER3, PRKG1 \\
\hline
\end{tabular}

KEGG, Kyoto Encyclopedia of Genes and Genomes; ECM, epithelial-mesenchymal transition.

Table III. Enriched pathways in modules.

\begin{tabular}{llll}
\hline Module & \multicolumn{1}{c}{ Gene set } & FDR & Nodes \\
\hline 1 & ECM-receptor interaction & $1.94 \times 10^{-12}$ & $\begin{array}{l}\text { GOLM1, MIA3, LAMA5, ITGA9, LAMB2, ITGB6, } \\
\text { MGAT4A, QSOX1, ITGB4, MFGE8, COL6A1, GAS6, } \\
\text { LAMA2, ADAM10 }\end{array}$ \\
& & & \\
& Focal adhesion & $3.88 \times 10^{-10}$ & \\
& PI3K-Akt signaling pathway & $9.34 \times 10^{-09}$ & \\
PPAR signaling pathway & $6.92 \times 10^{-08}$ & ACLY, ACSL1, CPT2, ACOX1, ACACB, FASN, SCD \\
& AMPK signaling pathway & $3.16 \times 10^{-05}$ & \\
Metabolic pathways & $6.41 \times 10^{-05}$ & \\
Pathways in cancer & $3.63 \times 10^{-05}$ & GNB2, ADCY5, ANAPC5, BUB3, CASR, NDC80, PLK1, \\
& Gap junction & $1.4 \times 10^{-04}$ & VHL, RNF144B, AREL1, PTGER3, NDE1, GPSM1, TRIM36, \\
& Cell cycle & FBXW7, LPAR1, ADCY9, CKAP5, MKRN1, BDKRB2 \\
\hline
\end{tabular}

FDR, false discovery rate; ECM, epithelial-mesenchymal transition; PPAR, peroxisome proliferator-activated receptor.

Morpheus is a useful online tool for analyzing gene expression that could be used to select DEGs (16). Therefore,
GSE103512 was downloaded and analyzed by Morpheus in the present study. The results showed that 2,000 DEGs were 

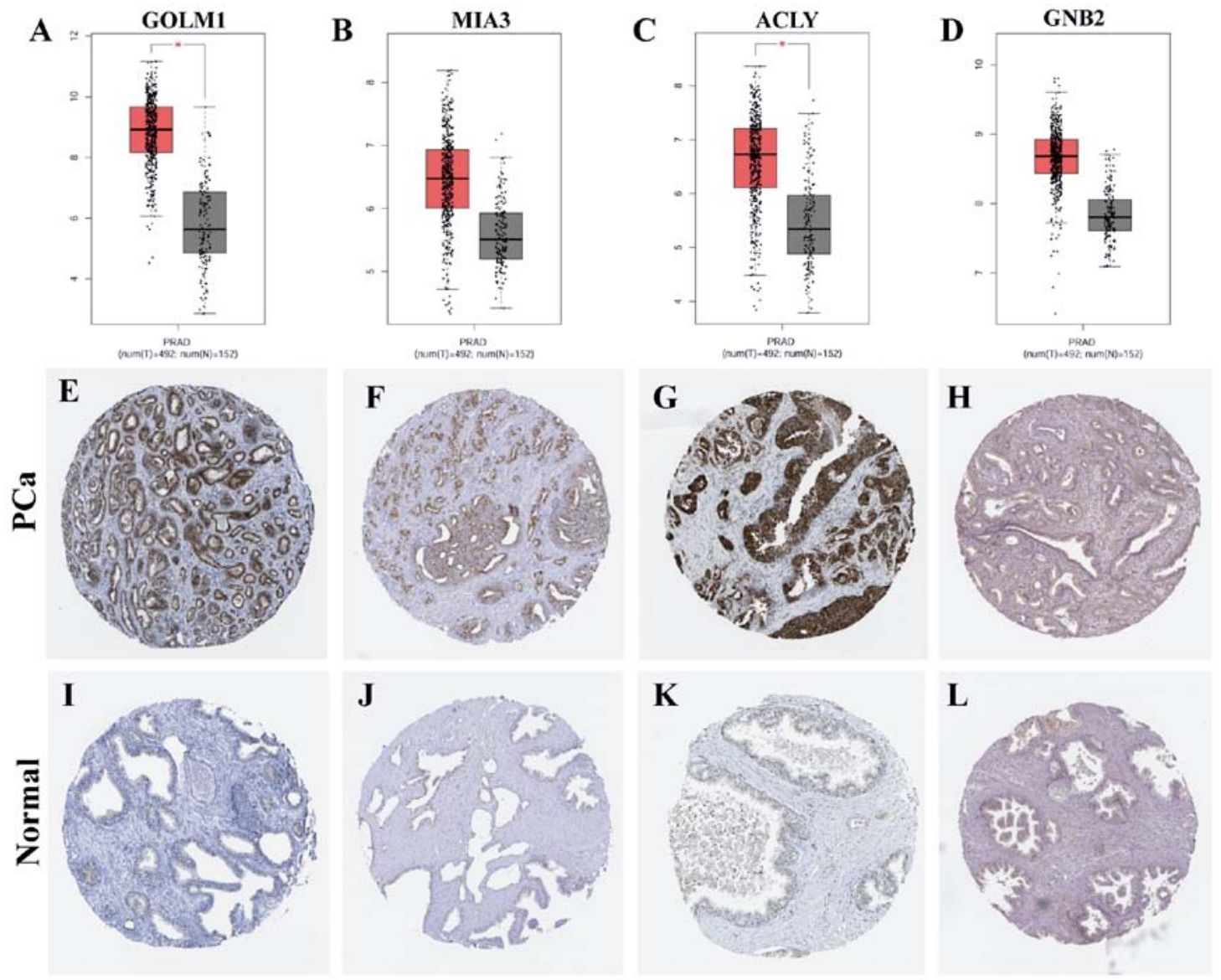

Figure 3. Expression of key genes in prostate cancer and normal prostate tissues. (A-D) The mRNA expression of the key genes was obtained from GEPIA (http://gepia.cancer-pku.cn/), including (A) GOLM1 (http://gepia.cancer-pku.cn/detail.php?gene=GOLM1), (B) MIA3 (http://gepia.cancer-pku.cn/detail. php?gene=MIA3), (C) ACLY (http://gepia.cancer-pku.cn/detail.php?gene=ACLY) and (D) GNB2 (http://gepia.cancer-pku.cn/detail.php?gene=GNB2). Red data columns represent prostate cancer; black data columns represent normal prostate tissues; T, tumor; N, normal. (E-L) Immunohistochemistry images of protein expression of genes were obtained from the Human Protein Atlas (https://www.proteinatlas.org/), including (E) GOLM1 (https://www.proteinatlas. org/ENSG00000135052-GOLM1/pathology/tissue/prostate+cancer\#img),(F)MIA3(https://www.proteinatlas.org/ENSG00000154305-MIA3/pathology/tissue/prostate+cancer\#img), (G) ACLY (https://www.proteinatlas.org/ENSG00000131473-ACLY/pathology/tissue/prostate+cancer\#img) and (H) GNB2 (https://www.proteinatlas.org/ENSG00000172354-GNB2/pathology/tissue/prostate+cancer\#img) in prostate cancer tissues, and (I) GOLM1 (https://www.proteinatlas. org/ENSG00000135052-GOLM1/tissue/prostate),(J)MIA3(https://www.proteinatlas.org/ENSG00000154305-MIA3/tissue/prostate),(K)ACLY(https://www. proteinatlas.org/ENSG00000131473-ACLY/tissue/prostate) and (L) (https://www.proteinatlas.org/ENSG00000172354-GNB2/tissue/prostate) in control tissues. Magnification, $\mathrm{x} 40 .{ }^{~} \mathrm{P}<0.05$. PRAD, prostate cancer; control, normal prostate tissues; GOLM1, Golgi membrane protein 1; MIA3, melanoma inhibitory activity member 3 ; ACLY, ATP citrate lyase; GNB2, G protein subunit $\beta 2$.
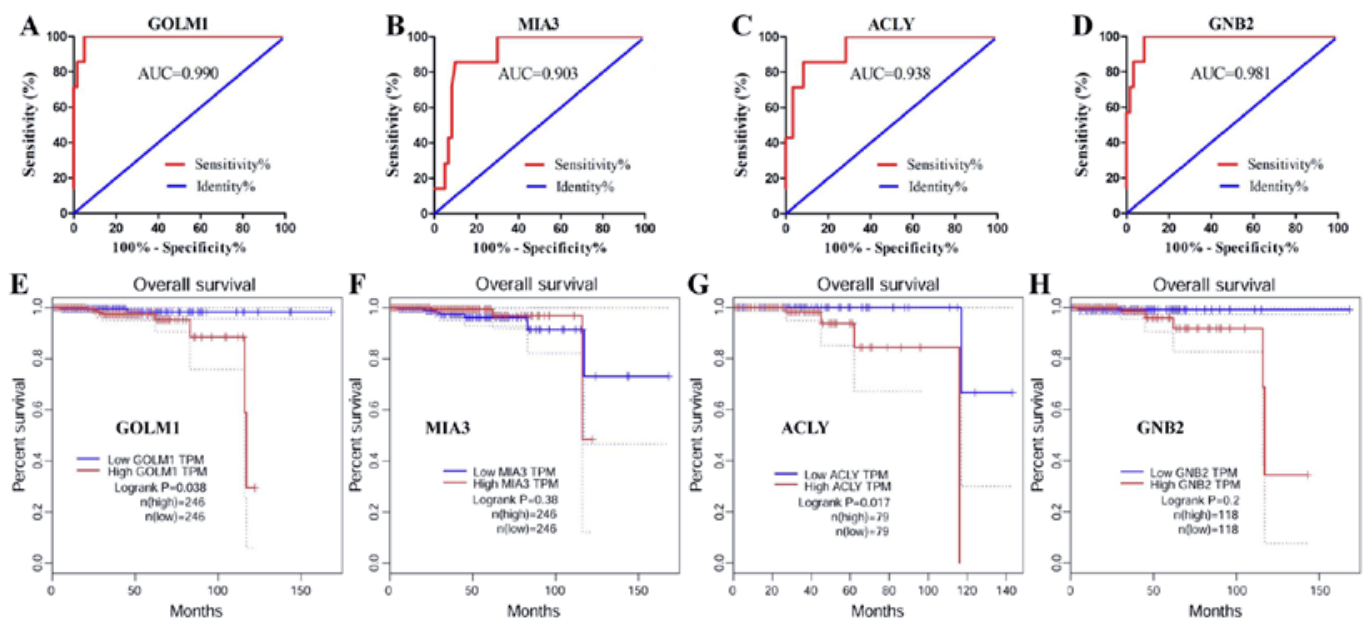

Figure 4. Identification of key genes for the diagnosis and prognosis of PCa. ROC analysis of the top four key genes was performed in PCa and normal prostate tissues. Survival curves were prepared for patients with PCa based on GEPIA data. ROC curve of (A) GOLM1, (B) MIA3, (C) ACLY and (D) GNB2 genes. Survival curve of (E) GOLM1, (F) MIA3, (G) ACLY and (H) GNB2. AUC, area under the curve; PCa, prostate cancer; control, normal prostate tissues; GOLM1, Golgi membrane protein 1; MIA3, melanoma inhibitory activity member 3; ACLY, ATP citrate lyase; GNB2, G protein subunit $\beta 2$; ROC, receiver operating characteristics; GEPIA, Gene Expression Profiling Interactive Analysis. 
screened, including 1,000 upregulated and 1,000 downregulated genes, in PCa compared with normal prostate tissues. A previous study has revealed that co-expressed genes are associated by similar biological function and signaling pathways (9). As a result, GO and KEGG analysis was further performed.

GO analysis indicated that the DEGs were mostly enriched in 'carboxylic acid catabolic process', 'cell apoptosis', 'cell proliferation' and 'cell migration' (Table I). These results indicated that the dysregulation of cell proliferation and cell migration was an important factor in the occurrence and metastasis of cancer $(19,20)$. KEGG analysis indicated that the DEGs were mostly concentrated in 'metabolic pathways', 'ECM-receptor interaction', the 'PI3K-Akt pathway' and 'focal adhesion' (Table II). Previous studies reported that cancer metabolism has emerged as an indispensable process of tumorigenesis $(21,22)$. Recent studies demonstrated that 'ECM-receptor interaction' and 'focal adhesion' were associated with tumor occurrence and metastasis $(23,24)$. Furthermore, another study implied that the 'PI3K-Akt pathway' served an important role in $\mathrm{PCa}$ progression (25).

PPI analysis was also performed in the present study and the following key genes with high degrees of interaction were found: GOLM1, MIA3, ACLY and GNB2. GOLM1 was the first identified key gene, and its functional role and molecular mechanism are unclear. Consistent with the present study, the study by Varambally et al (26) also reported that GOLM1 was upregulated in PCa tissues. It was further reported that GOLM1 may be secreted out of the cell by exosomes, which could be tested in the urine of patients with PCa (26). As a result, GOLM1 may act as a potential diagnostic biomarker of PCa. In addition, Yan et al (1) reported that GOLM1 could promote $\mathrm{PCa}$ progression by activating the PI3K-Akt pathway. Other studies also demonstrated that GOLM1 acted as a pivotal oncogene, involved in tumor cell migration and invasion in esophageal cancer (27) and hepatocellular carcinoma (28). These data suggested that GOLM1 may serve as a potential therapeutic target.

The second key gene identified in the present study was MIA3, which is an endoplasmic reticulum-resident protein (29). To the best of our knowledge, the study of MIA3 in cancer is limited. Arndt and Bosserhoff (30) reported that MIA3 may serve as a tumor suppressor of malignant melanoma. Gao et al (29) reported that miR-222 promoted migration through MIA3 in colorectal cancer cells. In the present study, the expression of MIA3 was elevated, which indicated that it may serve different functions in PCa compared with other tumors. The relevant underlying mechanism, however, requires further examination.

The third identified key gene was ACLY, which has been suggested to catalyze the formation of acetyl-CoA (31). To the best of our knowledge, ACLY has not been reported in $\mathrm{PCa}$, but has been reported in other types of tumors. A previous study reported that ACLY serves a critical role in tumorigenesis, and downregulation of ACLY expression may inhibit tumor cell proliferation in numerous types of cancer, such as lung, breast and bladder cancer (32). In addition, other studies further reported that inhibition of ACLY reversed the process of epithelial-mesenchymal transition in bronchial epithelial cells and non-small cell lung carcinoma cells $(31,33)$. Therefore, it was speculated that ACLY may serve as a key oncogene promoting the occurrence and metastasis of PCa.

The fourth identified key gene was GNB2, a member of the guanine nucleotide-binding proteins family. To the best of our knowledge, the related studies of GNB2 in cancer were limited. Kotani et al (34) reported that both mutation and overexpression of GNB2 could induce leukemogenesis, and it was indicated that the decrease of GNB2 expression reduced tumor cell proliferation. Akinori et al (35) reported that GNB2 mutations can activate the canonical signaling pathway and confer resistance to targeted kinase inhibitors in numerous types of cancer, such as acute myeloid leukemia and melanoma. To the best of our knowledge, the present study is the first to suggest that GNB2 was involved in PCa; however, the underlying mechanism requires further investigation. In addition, module analysis of PPI revealed that the genes in the three modules were associated with 'ECM-receptor interaction', 'focal adhesion', 'PI3K-Akt pathway', 'AMPK pathway', 'metabolic pathway', 'pathways in cancer', 'gap junction' and 'cell cycle'. These results were consistent with the GO and KEGG analysis.

GEPIA is a newly developed database including the data from The Cancer Genome Atlas and Genotype Tissue Expression projects (17). HPA is also a useful database for analysis of protein expression of genes between cancer tissues and normal tissues based on IHC (17). In the present study, GEPIA and HPA were used to identify the expression of key genes. The results showed that the expression of MIA3 and GNB2 was insignificantly upregulated in PCa compared with normal prostate tissues, and GOLM1 and ACLY expression was upregulated significantly. These results were matched with the bioinformatics analysis.

ROC and survival analyses were performed to evaluate the potential efficiency of these key genes as diagnostic and prognostic biomarkers. The results of ROC analysis showed that the AUC values of these genes were all $>0.85$. An AUC value $>0.5$ suggested the matter measured would yield significant distinction between the two groups $(36,37)$. Therefore, the results indicated that GOLM1, MIA3, ACLY and GNB2 had high sensitivity and specificity, and that they may be used as biomarkers for PCa diagnosis. The result of the survival analysis showed that high expression of GOLM1 and ACLY were associated with poor overall survival in patients with $\mathrm{PCa}$, which indicated that these genes may serve as candidate biomarkers for the prognosis of PCa.

In summary, this study identified GOLM1 and ACLY in $\mathrm{PCa}$, which may serve as potential diagnostic and prognostic biomarkers of PCa. It should be noted that this research was based on bioinformatics analysis. Therefore, relevant clinical studies are required, such as detection of the expression of the aforementioned genes in circulating tumor cells. In addition, the molecular mechanism of these genes will also be further studied to elucidate their role in the occurrence and metastasis of $\mathrm{PCa}$.

\section{Acknowledgements}

Not applicable. 


\section{Funding}

The study was supported by the Natural Science Foundation of Shandong Province (grant nos. ZR2014CL034 and ZR2018MC015), the Medical and Health Development Plan of Shandong Province (grant no. 2017WS058) and the Research and Development Plan of University in Shandong Province (grant no. J18KA120).

\section{Availability of data and materials}

All data generated or analyzed during this study are included in this published article. The mRNA expression profile of the GSE103512 dataset was downloaded from Gene Expression Omnibus (GEO) (http://www.ncbi.nlm.nih.gov/geo).

\section{Authors' contributions}

WF, ZFP and ZG designed the experiments and wrote the paper. QZ, XY, ZWP, YC, SH and GG analyzed the data.. All authors read and approved the final manuscript.

\section{Ethics approval and consent to participate}

Not applicable.

\section{Patient consent for publication}

Not applicable.

\section{Competing interests}

The authors declare that they have no competing interests.

\section{References}

1. Yan G, Ru Y, Wu K, Yan F, Wang Q, Wang J, Pan T, Zhang M, Han H, Li X and Zou L: GOLM1 promotes prostate cancer progression through activating PI3K-AKT-mTOR signaling. Prostate 78: 166-177, 2018.

2. Zhao H, Zhao X, Lei T and Zhang M: Screening, identification of prostate cancer urinary biomarkers and verification of important spots. Invest New Drugs, Jan 4, 2019 (Epub ahead of print).

3. Siegel RL, Miller KD and Jemal A: Cancer statistics, 2018. CA Cancer J Clin 68: 7-30, 2018.

4. Kudryavtseva AV, Lukyanova EN, Kharitonov SL, Nyushko KM, Krasheninnikov AA, Pudova EA, Guvatova ZG, Alekseev BY, Kiseleva MV, Kaprin AD, et al: Bioinformatic identification of differentially expressed genes associated with prognosis of locally advanced lymph node-positive prostate cancer. J Bioinform Computat Biol 17: 1950003, 2019.

5. Gadzinski AJ and Cooperberg MR: Prostate cancer markers Cancers Treat Res 175: 55-86, 2018.

6. Fujita $\mathrm{K}$ and Nonomura N: Urinary biomarkers of prostate cancer. Int J Urol 25: 770-779, 2018.

7. Thompson IM, Pauler DK, Goodman PJ, Tangen CM, Lucia MS, Parnes HL, Minasian LM, Ford LG, Lippman SM, Crawford ED, et al: Prevalence of prostate cancer among men with a prostate-specific antigen level $<$ or $=4.0 \mathrm{ng}$ per milliliter. N Engl J Med 350: 2239-2246, 2004.

8. Kulasingam V and Diamandis EP: Strategies for discovering novel cancer biomarkers through utilization of emerging technologies. Nat Clin Pract Oncol 5: 588-599, 2008.

9. Liang $\mathrm{B}, \mathrm{Li} \mathrm{C}$ and Zhao J: Identification of key pathways and genes in colorectal cancer using bioinformatics analysis. Med Oncol 33: 111, 2016
10. Lascorz J, Hemminki K and Försti A: Systematic enrichment analysis of gene expression profiling studies identifies consensus pathways implicated in colorectal cancer development. J Carcinog 10: 7, 2011

11. Cheng Y, Wang K, Geng L, Sun J, Xu W, Liu D, Gong S and Zhu Y: Identification of candidate diagnostic and prognostic biomarkers for pancreatic carcinoma. EBioMedicine 40: 382-393, 2019.

12. Shinichi Y, Sian J, Ivana B, Antal T, Leary R, Fu B, Kamiyama M, Hruban RH, Eshleman JR, Nowak MA, et al: Distant metastasis occurs late during the genetic evolution of pancreatic cancer. Nature 467: 1114-1117, 2010.

13. Brouwer-Visser J, Cheng WY, Bauer-Mehren A, Maisel D, Lechner K, Andersson E, Dudley JT and Milletti F: Regulatory T-cell genes drive altered immune microenvironment in adult solid cancers and allow for immune contextual patient subtyping. Cancer Epidemiol Biomarkers Prev 27: 103-112, 2018.

14. Mi B, Liu G, Zhou W, Lv H, Liu Y and Liu J: Identification of genes and pathways in the synovia of women with osteoarthritis by bioinformatics analysis. Mol Med Rep 17: 4467-4473, 2018.

15. Franceschini A, Szklarczyk D, Frankild S, Kuhn M, Simonovic M, Roth A, Lin J, Minguez P, Bork P, von Mering C and Jensen LJ: STRING v9.1: Protein-protein interaction networks, with increased coverage and integration. Nucleic Acids Res 41 (Database Issue): D808-D815, 2013.

16. Fan S, Liang Z, Gao Z, Pan Z, Han S, Liu X, Zhao C, Yang W, Pan $\mathrm{Z}$ and Feng W: Identification of the key genes and pathways in prostate cancer. Oncol Lett 16: 6663-6669, 2018.

17. Zhang B, Wu Q, Wang Z, Xu R, Hu X, Sun Y, Wang Q, Ju F, Ren S, Zhang C, et al: The promising novel biomarkers and candidate small molecule drugs in kidney renal clear cell carcinoma: Evidence from bioinformatics analysis of high $\square$ throughput data. Mol Genet Genomic Med 7: e607, 2019.

18. Jin Q, Dai Y, Wang Y, Zhang S and Liu G: High kinesin family member 11 expression predicts poor prognosis in patients with clear cell renal cell carcinoma. J Clin Pathol 72: 354-362, 2019

19. Perez R, Wu N, Klipfel AA and Beart RW Jr: A better cell cycle target for gene therapy of colorectal cancer: Cyclin G. J Gastroint Surg 7: 884-889, 2003

20. Tsunoda T, Nakamura T, Ishimoto $K$, Yamaue $H$, Tanimura $H$, Saijo $\mathrm{N}$ and Nishio K: Upregulated expression of angiogenesis genes and down regulation of cell cycle genes in human colorectal cancer tissue determined by cDNA macroarray. Anticancer Res 21: 137-143, 2001.

21. Dong W, Keibler MA and Stephanopoulos G: Review of metabolic pathways activated in cancer cells as determined through isotopic labeling and network analysis. Metab Eng 43: 113-124, 2017.

22. Zarrinpar A: Metabolic pathway inhibition in liver cancer. SLAS Technol 22: 237-244, 2017.

23. Zhang HJ, Tao J, Sheng L, Hu X, Rong RM, Xu M and Zhu TY: Twist 2 promotes kidney cancer cell proliferation and invasion by regulating ITGA6 and CD44 expression in the ECM-receptor interaction pathway. OncoTargets Ther 9: 1801-1812, 2016.

24. Eke I and Cordes N: Focal adhesion signaling and therapy resistance in cancer. Semin Cancer Biol 31: 65-75, 2015.

25. Yang L, Zha TQ, He X, Chen L, Zhu Q, Wu WB, Nie FQ, Wang Q, Zang CS, Zhang ML, et al: Placenta-specific protein 1 promotes cell proliferation and invasion in non-small cell lung cancer. Oncol Rep 39: 53-60, 2018.

26. Varambally S, Laxman B, Mehra R, Cao Q, Dhanasekaran SM, Tomlins SA, Granger J, Vellaichamy A, Sreekumar A, Yu J, et al: Golgi protein GOLM1 is a tissue and urine biomarker of prostate cancer. Neoplasia 10: 1285-1294, 2008.

27. Byrne AM, Bekiaris S, Duggan G, Prichard D, Kirca M, Finn S, Reynolds JV, Kelleher D and Long A: Golgi phosphoprotein 2 (GOLPH2) is a novel bile acid-responsive modulator of oesophageal cell migration and invasion. Br J Cancer 113: 1332-1342, 2015.

28. Ye QH, Zhu WW, Zhang JB, Qin Y, Lu M, Lin GL, Guo L, Zhang B, Lin ZH, Roessler S, et al: GOLM1 modulates EGFR/RTK cell-surface recycling to drive hepatocellular carcinoma metastasis. Cancer Cell 30: 444-458, 2016.

29. Gao H, Cong X, Zhou J and Guan M: MicroRNA-222 influences migration and invasion through MIA3 in colorectal cancer. Cancer Cell Int 17: 78-87, 2017.

30. Arndt S and Bosserhoff AK: TANGO is a tumor suppressor of malignant melanoma. Int J Cancer 119: 2812-2820, 2010. 
31. Fu Y, Lu R, Cui J, Sun H, Yang H, Meng Q, Wu S, Aschner M, Li X and Chen R: Inhibition of ATP citrate lyase (ACLY) protects airway epithelia from $\mathrm{PM}_{2.5}$-induced epithelial-mesenchymal transition. Ecotoxicol Environmen Saf 167: 309-316, 2019.

32. Icard $\mathrm{P}$ and Lincet $\mathrm{H}$ : The reduced concentration of citrate in cancer cells: An indicator of cancer aggressiveness and a possible therapeutic target. Drug Resist Updat 29: 47-53, 2016.

33. Hanai J, Doro N, Sasaki AT, Kobayashi S, Cantley LC, Seth P and Sukhatme VP: Inhibition of lung cancer growth: ATP citrate lyase knockdown and statin treatment leads to dual blockade of mitogen-activated protein kinase (MAPK) and phosphatidylinositol-3-kinase (PI3K)/AKT pathways. J Cell Physiol 227: 1709-1720, 2012.

34. Kotani S, Yoda A, Kon A, Kataoka K, Ochi Y, Shiozawa Y, Hirsch C, Takeda J, Ueno H, Yoshizato T, et al: Molecular pathogenesis of disease progression in MLL-rearranged AML. Leukemia 33: 612-624, 2019.
35. Yoda A, Adelmant G, Tamburini J, Chapuy B, Shindoh N, Yoda Y, Weigert O, Kopp N, Wu SC, Kim SS, et al: Mutations in $\mathrm{G}$ protein $\beta$ subunits promote transformation and kinase inhibitor resistance. Nat Med 21: 71-75, 2015.

36. Arthurs C, Murtaza BN, Thomson C, Dickens K, Henrique R, Patel HRH, Beltran M, Millar M, Thrasivoulou C and Ahmed A: Expression of ribosomal proteins in normal and cancerous human prostate tissue. PLoS One 12: e0186047, 2017.

37. Fawcett T: An introduction to ROC analysis. Pattern Recog Lett 27: 861-874, 2006.

(c) (i) () (9) This work is licensed under a Creative Commons c) Attribution-NonCommercial-NoDerivatives 4.0 International (CC BY-NC-ND 4.0) License. 